\title{
OB stars towards NGC 6357 and NGC 6334
}

\section{Delphine Russeil}

Aix Marseille Univ, CNRS, LAM, Laboratoire d'Astrophysique de Marseille, Marseille, France email: delphine.russeil@lam.fr

\begin{abstract}
The star forming regions NGC6334 and NGC6357 are amid the most active starforming complexes of our Galaxy where massive star formation is occuring. Both complexes gather several HII regions but they exhibit different aspects: NGC6334 is characterised by a dense molecular ridge where recent massive star formation is obvious while NGC6357 is dominated by the action of the stellar cluster Pismis 24 which have shaped a large cavity. To understand and compare the formation of massive stars in these two regions requires to precise the distance and characterise the proper motions of the $\mathrm{O}$ to B3 stellar population in these regions.
\end{abstract}

Keywords. stars: distances, stars: early-type, ISM: HII regions

\section{Overview and GAIA perspectives}

The NGC6334 and NGC6357 (Fig. 1a) are two active star-forming complexes for which it is possible to study the formation and the evolution of massive stars from their earliest phase to their impact on the environment due to their HII regions. From the OB stars distribution, mainly distributed between 1 and $3 \mathrm{kpc}$ (Russeil et al. 2012), a distance of $1.75 \mathrm{kpc}$ for both regions is usualy adopted. From the GAIA-DR1 catalogue (e.g. Arenou et al. 2017, Lindegren et al. 2016) one can underline (Fig. 1b and c) a stellar layer around $1.12 \mathrm{kpc}$ (main peak of the histogram Fig. 1c at $\sim 0.89$ mas) while few O-B3 stars towards NGC 6334 and NGC 6357 have a measured parallactic distance (see Table 1) in the GAIA DR1 catalogue (Astraatmadja et al. 2016). Despite our small sample the mean distance obtained is $2.23 \mathrm{kpc}$ or $1.23 \mathrm{kpc}$ depending on the prior. The Milky-way prior gives better agreement with Wu et al. 2014 and Chibueze et al. 2014 who gives for NGC 6334 a maser parallax distance between 1.25 and $1.35 \mathrm{kpc}$.

In parallel, WISE- $22 \mu \mathrm{m}$ and HERSCHEL-70 $\mu \mathrm{m}$ data were used to identify (Russeil et al. 2016) bow shocks features (Fig. 1d) which are usualy related to run-away stars (e.g. Gvaramadze et al. 2011). The identified features are either seen in $\mathrm{H} \alpha$ or radio continuum suggesting that run-away stars can be massive.

In addition, an age estimate of O-B3 stars (Russeil et al., in preparation) suggest that young $(\log ($ age $)<7)$ stars are found at the periphery of NGC 6357 . They can have been formed in Pismis 24 but quickly expelled.

Table 1. O-B3 stars with parallax distance in NGC 6334 and NGC 6357.

\begin{tabular}{|l|l|l|l|l|l|}
\hline HII region & Star Ident. & Spectral type & $\begin{array}{l}\text { Dist. } \\
\text { kpc }\end{array}$ & $\begin{array}{l}\text { G AIA dist. } \\
\text { kpc }\end{array}$ & $\begin{array}{l}\text { GAIA dist. } \\
\text { kpc }\end{array}$ \\
\hline NGC 6334 - GUM64C & CD-3511482 & B0.5e & 1.41 & $2.71 \pm 2.20$ & $1.21 \pm 0.43$ \\
NGC 6334 - GUM64b & HD319702 & O8III & 2.11 & $1.66 \pm 1.82$ & $1.17 \pm 0.36$ \\
NGC 6334 - GUM64b & CD-3511484 & B1V & 1.10 & $2.10 \pm 1.93$ & $1.25 \pm 0.38$ \\
NGC 6334 - G351.2+0.5 & HD156738 & O6.6III(f) & 1.58 & $2.67 \pm 2.06$ & $1.31 \pm 0.40$ \\
\hline NGC 6357 & HD157504 & WN5b & - & $2.75 \pm 2.13$ & $1.26 \pm 0.39$ \\
NGC 6357 & HD319881 & O6Vn & 1.66 & $1.49 \pm 1.73$ & $1.19 \pm 0.50$ \\
\hline
\end{tabular}

Notes: ${ }^{1}$ Spectro-photometric distance (Russeil et al. 2012). ${ }^{2}$ Distances from Gaia DR1 catalogue parallaxes computed by Astraatmadja et al. 2016 with the exponential decreasing space density prior with $\mathrm{L}=1.35 \mathrm{kpc}$ (systematics uncertainties of 0.3 mas included). ${ }^{3}$ Same as ${ }^{2}$ but using the Milky-Way prior. 

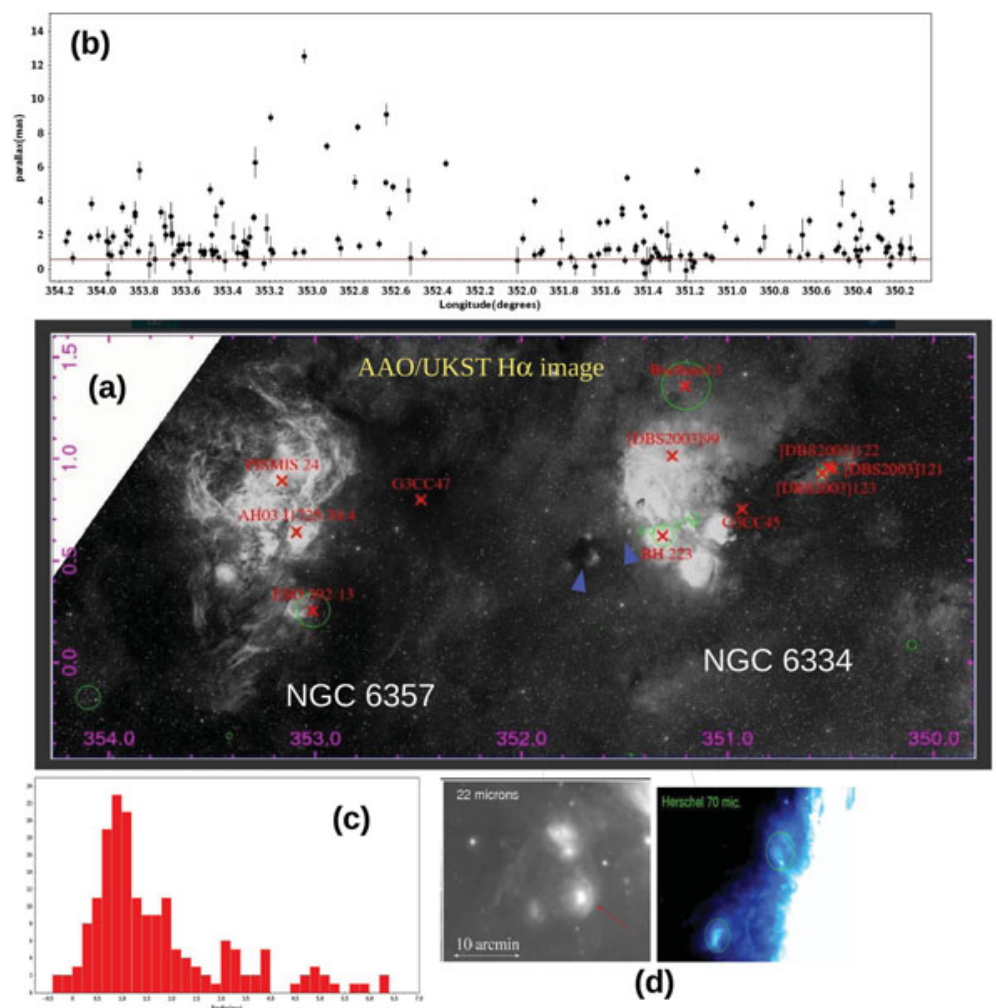

(d)

Figure 1. (a) $\mathrm{H} \alpha$ image of NGC 6334 and NGC 6357. (b) GAIA DR1 sources $\left(0.5^{\circ}<\mathrm{b}<\right.$ $1.5^{\circ}$ ) parallaxes (histogram in (c)) versus longitude (the horizontal line underlines the $1.75 \mathrm{kpc}$ parallax). (d) Candidate run-away stars identified by Russeil et al. 2016.

To summarise, the next GAIA release will be used to 1) determine/precise statistically the distance of the $\mathrm{OB}$ stars and the different stellar layers present along the line of sight of NGC 6334 and NGC 6357, 2) identify and precise the proper motion of the run-away stars in order to clarify their origin and their possible star-formation impact.

\section{Acknowledgements}

This work has made use of data from the European Space Agency (ESA) mission Gaia (https://www. cosmos.esa.int/gaia), processed by the Gaia Data Processing and Analysis Consortium (DPAC, https://www.cosmos.esa.int/web/gaia/dpac/ consortium). Funding for the DPAC has been provided by national institutions, in particular the institutions participating in the Gaia Multilateral Agreement.

\section{References}

Arenou, et al. 2017, Aछ A, 599, A50

Astraatmadja, et al. 2016, ApJ, 833, 119

Chibueze, et al. 2014, ApJ, 784, 114

Gvaramadze, et al. 2011, A\&\&A, 535, A29

Lindegren, et al. 2016, A\&A, 595, A4

Russeil, et al. 2012, A\& A, 538, A142

Russeil, et al. 2016, A\&SA, 587, A135

$\mathrm{Wu}$, et al. 2014, Aध่A, 566, A17 